\title{
Database for LDV Signal Processor Performance Analysis
}

Glenn D. Baker, R. Jay Murphy

Macrodyne, Inc.

Clifton Park, New York

and

James F. Meyers

NASA Langley Research Center Hampton, Virginia

13th International Congress on Instrumentation in Aerospace Simulation Facilities

September 18-21, 1989

Göttingen, West Germany 



\title{
Database for LDV Signal Processor Performance Analysis
}

\author{
by \\ Glenn D. Baker and R. Jay Murphy \\ Macrodyne, Inc. \\ Clifton Park, New York, USA \\ and \\ James F. Meyers \\ NASA - Langley Research Center \\ Hampton, Virginia, USA
}

\begin{abstract}
A comparative and quantitative analysis of various laser velocimeter signal processors is difficult because standards for characterizing signal bursts have not been established. This leaves the researcher to select a signal processor based only on manufacturers' claims without the benefit of direct comparison. The present paper proposes the use of a database of digitized signal bursts obtained from a laser velocimeter under various configurations as a method for directly comparing signal processors.
\end{abstract}

\section{Introduction}

A number of techniques have been developed since the advent of laser velocimetry in 1964 [1] to measure the frequency within a signal burst obtained as a small particle passes through the fringe pattern. These techniques include spectrum analyzers, wide band frequency trackers [2-3], high-speed burst counters [4-6], photon correlators [7], filter banks and recently, digital FFT signal processors [8-9]. Within each of these techniques, many approaches have been implemented by various manufacturers. Each has claimed superiority of their chosen technique and in particular, their approach to implementing that technique. It is difficult for the researcher to decipher these various biased claims to determine which manufacturer has the best signal processor for his application.

This problem would be minimized if the researcher had access to the various signal processors. A direct comparison could then be conducted to determine which was the best solution for his application. The 
obvious comparative testing scheme would be to input signals from a laser velocimeter simultaneously to the various signal processors. The unit with the best characteristics and the greatest accuracy would be the processor of choice. However, this approach contains a flaw in that the true signal frequencies are not known. Therefore measurement accuracies and even instrumentation characteristics can not be reliably determined.

The next logical approach would be to use signals with known frequencies from sine wave generators. The researcher may add Gaussian noise or even use these signals to drive a light emitting diode to better simulate the passage of the particle through the fringe pattern. Problems with this approach include the introduction of simplifying assumptions, process dependent variables, elimination of burst capture variables, and unintentional bias on the part of the researcher in establishing the signal characteristics.

With the realization that actual bursts arrive at random times and are composed of randomly occurring photons resulting in random changes in the burst characteristics, it becomes obvious that signal generators can not be used as simulators. The next approach would be the use of random number generators in a computer to build simulated signals [10]. While this approach has many advantages and provides solutions to many of the problems with the previous techniques, it still suffers from process dependent variables and unintentional bias on the part of the researcher.

Most likely, our example researcher has long ago given up and purchased a unit based on such scientific criteria as lowest price, slick advertising, or promises of greatness from the manufacturer. If, however, our researcher had used a high-speed transient recorder to digitize the signal bursts from the laser velocimeter, their true signal frequencies could be determined by computer analysis of the captured waveforms. This would result in defining a standard set of laser velocimeter signals which typify those expected from his system. This database could then be sent to various manufacturers for analysis. The present paper presents the procedure for acquiring and analyzing such a signal burst database.

\section{Signal Development}

The database should include signals representing all amplitudes and signal-to-noise ratios expected during normal operation of the laser velocimeter. The database should contain signal frequencies expected from the full velocity range and include Bragg shifting if used. If 
boundary layer measurements are expected, signals containing stray light from flare should be included. The present database includes signals representing all conditions since it is designed to approximate the expected signals from a generalized laser velocimeter.

The optical system was a standard one-component fringe type laser velocimeter configured in the backscatter mode. The receiver was placed 45 degrees off- axis in the plane of the two crossing laser beams, figure 1. The characteristics of the sample volume were varied by using transmitting lenses of different focal lengths. The short focal length, $0.37 \mathrm{~m}$, produced a sample volume with a calculated diameter of $0.2 \mathrm{~mm}$ and fringe spacing of $3.88 \mu \mathrm{m}$. The longer focal length, $1.2 \mathrm{~m}$, resulted in a calculated diameter of $0.65 \mathrm{~mm}$ and fringe spacing of $12.36 \mu \mathrm{m}$. The receiver had a $0.37 \mathrm{~m}$ focal length lens with a collecting aperture of $0.062 \mathrm{~m}$. The photomultiplier had a quantum efficiency at $514.5 \mathrm{~nm}$ of 0.21 and a measured gain (overall system transfer function based on a single input photon, figure 2) of approximately 3,200,000. A single Bragg cell provided frequency shifting. Simulation of background flare was obtained by placing a flat black aluminum block in one of the laser beams following crossover. The amount of diffuse scattered light entering the receiver was controlled by placement of the block along the beam.

The laser velocimeter sample volume was placed on the centerline in an empty test section of a small subsonic wind tunnel. Hot wire measurements of the free stream turbulence indicated intensities of 0.1 percent at $10 \mathrm{w}$ speed up to 0.2 percent at maximum velocity. Polystyrene particles, $0.8 \mu \mathrm{m}$ in diameter, provided maximum particle tracking fidelity in the flow and minimized variations in signal characteristics. A 50/50 mix of water and ethanol provided the carrier for the particles. The liquid evaporated within $20 \mathrm{~cm}$ of the aerosol nozzle leaving the dry particles to follow the air flow. The small variation in signal level and visual inspection of the captured signal bursts verify the lack of particle agglomeration.

The transient recorder was a Hewlett-Packard model 54111D digital storage oscilloscope. This unit has the capability of sampling at $1.0 \mathrm{GHz}$ with a single shot bandwidth of $250 \mathrm{MHz}$. The analog-to-digital converter has 8-bit resolution up to $25 \mathrm{MHz}$, 7-bit resolution to $100 \mathrm{MHz}$ and 6 -bit resolution beyond. The recorded traces are 8192 samples in length. The oscilloscope was manually adjusted to center the signal bursts within its storage record prior to acquisition by an IBM-AT compatible computer. The data ensemble of 128 captured signal bursts for each optical configuration was stored on hard disk. 


\section{Signal Processing}

The characteristics of each signal burst were determined and used to calculate the mean, standard deviation and distribution of each signal parameter within the data ensemble. These parameters include photon count, signal amplitude and visibility [11], signal-to-noise ratio, and signal frequency. The processing procedures can be illustrated by analyzing two example signal bursts. The first burst, figure 3, was obtained with the short focal length system, low laser power and minimal background light. The second, figure 4, was obtained with the long focal length system, medium laser power and background flare adding substantial noise to the signal.

The number of photons contained within a signal burst is estimated by normalizing the integral of that burst with the integral of the transfer function for a single photon. The length of a signal burst is obtained by truncating the waveform at the $1 / \mathrm{e}^{2}$ points corresponding to the generally accepted limits of the laser velocimeter sample volume. The integrals of the example signals, defined within the vertical lines in figures 3 and 4 , are $1.20 \times 10^{-7} \mathrm{~V}$-sec and $1.23 \times 10^{-7} \mathrm{~V}$-sec respectively. Since the single photon transfer function, figure 2, contains $1.638 \times 10^{-11} \mathrm{~V}$-sec, the example signal bursts were generated by 7341 photons and 7536 photons respectively.

The signal burst is then transformed to the frequency domain using complex Fourier transform algorithms for 8192 points. The power spectra is then calculated by summing the squares of the real and imaginary parts. This spectra, figures 5 and 6 , is used to determine the locations of the pedestal and signal power. The location of the pedestal is found by following the spectral curve until the slope becomes positive, indicated by the vertical line on the left of figures 5 and 6 . The location of the signal is determined in the same manner on both sides of the major peak to the right of the pedestal. Signal-to-noise can now be calculated by dividing the in tegral of the signal by the integral of the total spectra less the power contained within the pedestal and signal. For the present examples, figures 5 and 6 , the signal-to-noise ratios are $-2.52 \mathrm{~dB}$ and $-8.376 \mathrm{~dB}$ respectively.

The signal frequency is obtained by performing a bin omial regression fit of the five adjacent samples centered at the peak of the signal spectra, figures 5 and 6 . The two frequencies, $9.66 \mathrm{MHz}$ and $3.01 \mathrm{MHz}$ respectively, were found by determining the maxima of the binomial fits.

Estimates of signal amplitude and visibility should be based on signals without noise. The noise is removed by multiplying the complex spectra 
by brickwall filters shown as boxes surrounding the pedestal and signal components in figures 5 and 6 . After multiplication, the complex spectra is inverse transformed to yield the time domain signals in figures 7 and 8 . The peak amplitude is now easily determined. The visibility function [11] is defined as:

$$
\text { visibility }=\frac{V_{\max }-V_{\min }}{V_{\max }+V_{\min }}
$$

where the minimum, $V_{m i n}$, is defined as the voltage one half cycle from the peak voltage, $V_{\max }$. An alternative form of equation (1) is:

$$
\text { visibility }=\frac{P_{f}}{P_{t}-P_{f}}
$$

where $P_{t}$ is equal to the peak voltage, $V_{\max }$, and $P_{f}$ is the peak of the bandpass filtered signal, figures 9 and 10 . Thus the visibility of the two example signals are 0.88 and 0.83 respectively.

\section{The Example Database}

A database of laser velocimeter signal bursts has been developed using the approach presented previously. The database includes signal ensembles obtained with three laser power settings at two transmitted focal lengths. Data was acquired at two flow velocities yielding signal frequencies of $1.6 \mathrm{MHz}$ and $9.7 \mathrm{MHz}$ with the short focal length and $0.5 \mathrm{MHz}$ and $3.0 \mathrm{MHz}$ at the long focal length. The signals were sampled at $50 \mathrm{MHz}, 250 \mathrm{MHz}$ and $500 \mathrm{MHz}$. A Bragg cell was included for several ensembles to provide a frequency bias of $40 \mathrm{MHz}$. Bragg shifted signals downmixed to $2 \mathrm{MHz}$ and $10 \mathrm{MHz}$ are also included. These conditions were repeated with the inclusion of flare to decrease the signal-to-noise ratio. The resulting database contained signals with peak voltages from $0.022 \mathrm{~V}$ to $0.2 \mathrm{~V}$, visibilities from 0.61 to 0.82 , and signal-to-noise ratios from $-11.1 \mathrm{~dB}$ to $6.2 \mathrm{~dB}$.

The distribution of signal characteristics within an ensemble may be illustrated by collapsing the data to histograms. The characteristics of the ensembles obtained using the maximum output power, short focal length, and a frequency shift of $40 \mathrm{MHz}$ provide examples of this procedure. The distribution of peak signal amplitude is shown in figures 11 and 12. It is noted that the signal amplitude increased by approximately a factor of two when background flare was added. Comparisons of signal visibility, signal-to-noise and signal frequency are shown in figures 13 through 18 . Although the characteristics appear 
to be similar, views of two signal bursts, figures 19 and 20, appear to have a greater difference in quality than the 2 to $3 \mathrm{~dB}$ difference in signal-to-noise they actually have. Closer investigation of the signals reveal the presence of the $40 \mathrm{MHz}$ Bragg frequency and the $70 \mathrm{MHz}$ longitudinal mode frequency of the laser. Since these frequencies are coherent, it appears that they would be strong contributors to a reduction in signal-to-noise. Power spectra measurements of these signals however, do not show coherent spectral peaks from these frequency sources.

\section{Concluding Remarks}

A method for establishing a database of laser velocimeter signal bursts has been presented. This database can be used to evaluate the performance of signal processors and processor algorithms to specific signal characteristics and not to generalized simplistic waveforms. Examples from such a database have been presented to illustrate the capabilities of the proposed method.

\section{References}

[1] Yeh, Y. and Cummins, H. Z.: Localized Fluid Flow Measurements with an He-Ne Laser Spectrometer. Applied Physics Letters, vol. 4, pp. 176-178, May 1964.

[2] Huffaker, R. M.; Fuller, C. E.; and Lawrence, T. R.: Application of Laser Doppler Velocity Instrumentation to the Measurement of Jet Turbulence. Paper 690266, Soc. Automotive Engineers, January, 1969.

[3] Fridman, J. D.; Kinnard, K. F.; and Meister, K.: Laser Doppler Instrumentation for the Measurement of Turbulence in Gas and Fluid Flows. Proceedings of the Technical Program - ElectroOptical Systems Design Conference, Karl A. Kopetzky, ed., Industrial and Scientific Conference Management, Inc., c.1970, pp. 128-146.

[4] Asher, J. A.: LDV Systems Development and Testing. The Use of the Laser Doppler Velocimeter for Flow Measurements, W. H. Stevenson and H. D. Thompson, eds., Contract N00014-67-A0226055, Purdue Univ., Nov. 1972, pp. 338-347.

[5] Lennert, A. E.; Smith, F. H., Jr.; and Kalb, H. T.: Application of Dual Scatter Laser Doppler Velocimeters for Wind Tunnel 
Measurements. International Congress on Instrumentation in Aerospace Simulation Facilities - ICIASF '71 Record, IEEE Publ. 71-C-33 AES, Inst. of Electrical and Electronics Engineers, Inc., c.1971, pp. 157-167.

[6] Iten, P. D.; and Mastner, J.: Laser Doppler Velocimeter Offering High Spatial and Temporal Resolution. Flow - Its Measurement and Control in Science and Industry, Volume One, Part Two, R. E. Wendt, Jr., ed., Instrument Soc. of America, 1974, pp. 1007-1013.

[7] Pike, E. R.: The Application of Photon-Correlation Spectroscopy to Laser Doppler Velocimetry. The Use of the Laser Doppler Velocimeter for Flow Measurements, W. H. Stevenson and H. D. Thompson, eds., Contract N00014-67-A0226-055, Purdue Univ., Nov. 1972, pp. 338-347.

[8] Meyers, J. F.; and Stoughton, J. W.: Frequency Domain Laser Velocimeter Signal Processor. Third International Symposium on Applications of Laser Anemometry to Fluid Mechanics, Lisbon, Portugal, July 7-9, 1986.

[9] Lading, L.: Spectrum Analysis of LDA Signals. Symposium on the Use of Computers in Laser Velocimetry - Proceedings, SaintLouis, France, May, 1987.

[10] Mayo, W. T., Jr.: Modeling Laser Velocimeter Signals as Triply Stochastic Poisson Processes. Minnesota Symposium on Laser Anemometry - Proceedings, E.R.G. Eckert, ed., Univ. of Minnesota, January 1976, pp. 455-484.

[11] Farmer, W. M.: The Interferometric Observation of Dynamic Particle Size, Velocity, and Number Density. Ph.D. Thesis, Tennessee Univ., Knoxville, Tennessee, 1974. 


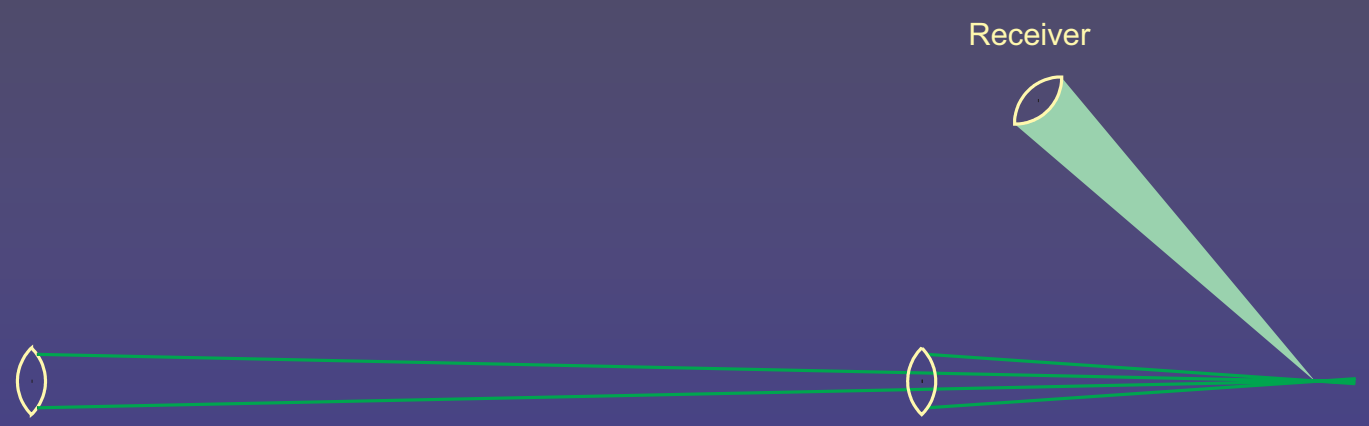

Lens position 2

Lens position 1

Figure 1.- Schematic of the laser velocimeter optical configuration.

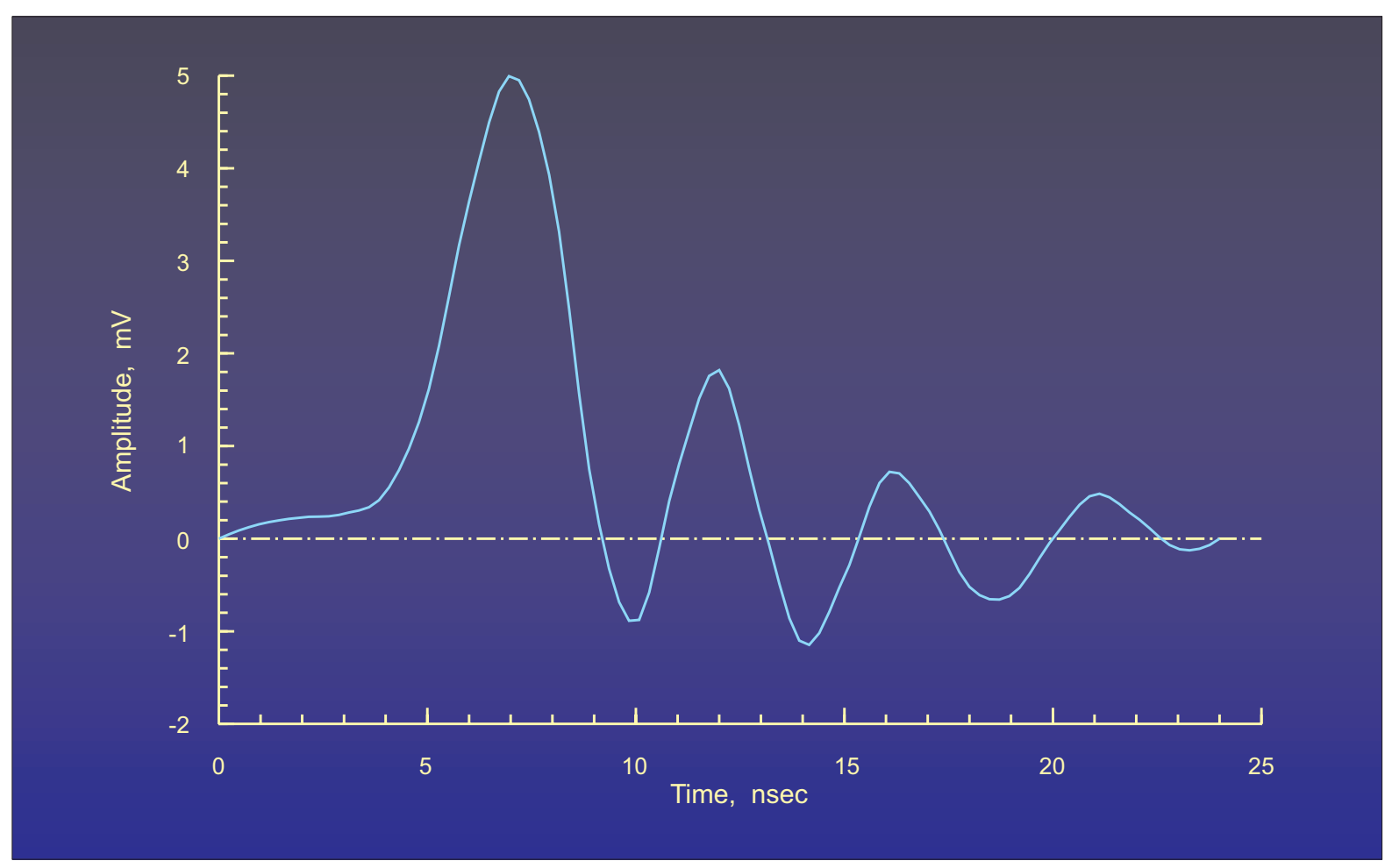

Figure 2.- Photomultiplier transfer function for a single photon. 


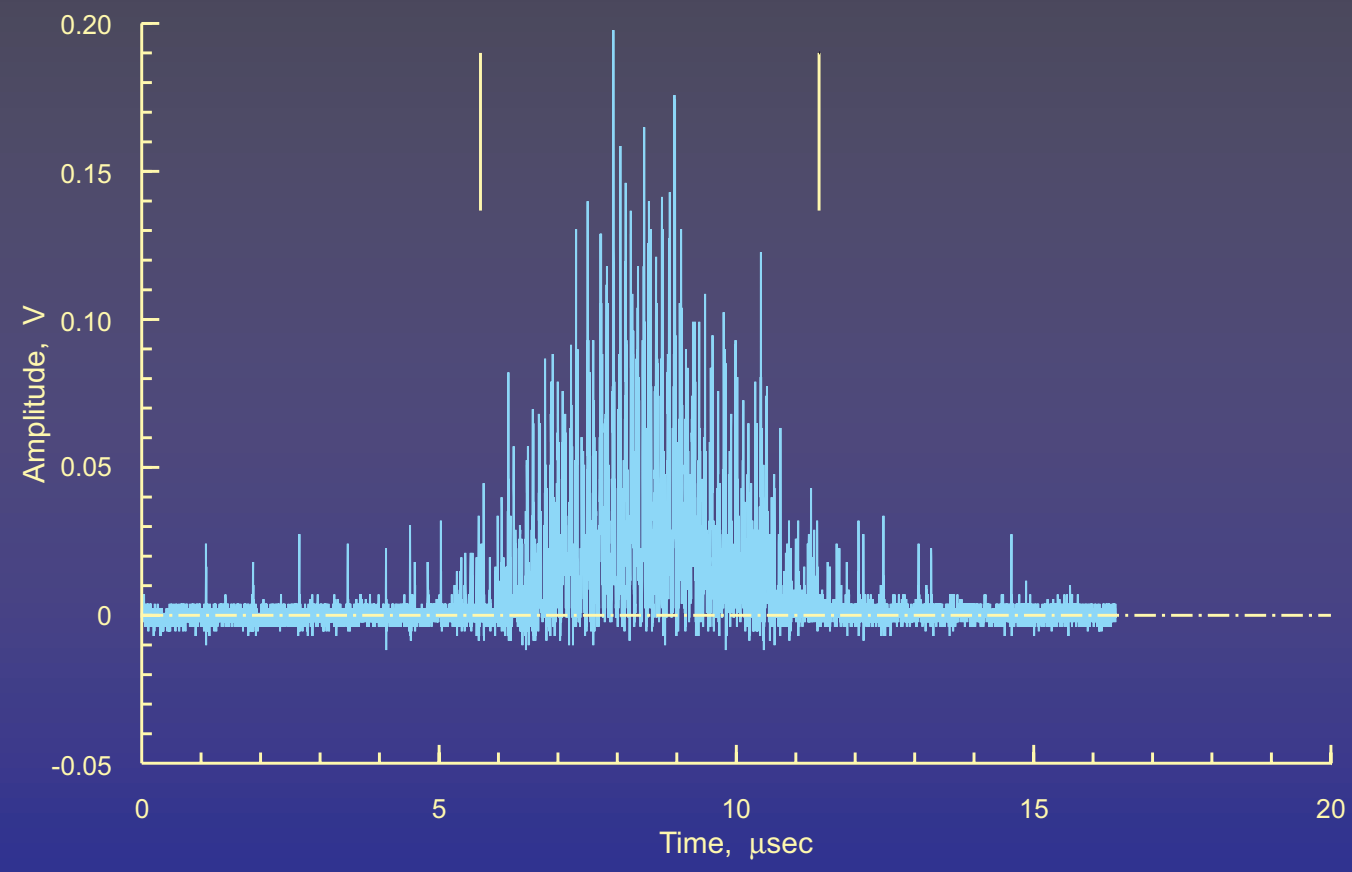

Figure 3.- Captured signal burst from the short focal length configuration with the low laser power setting.

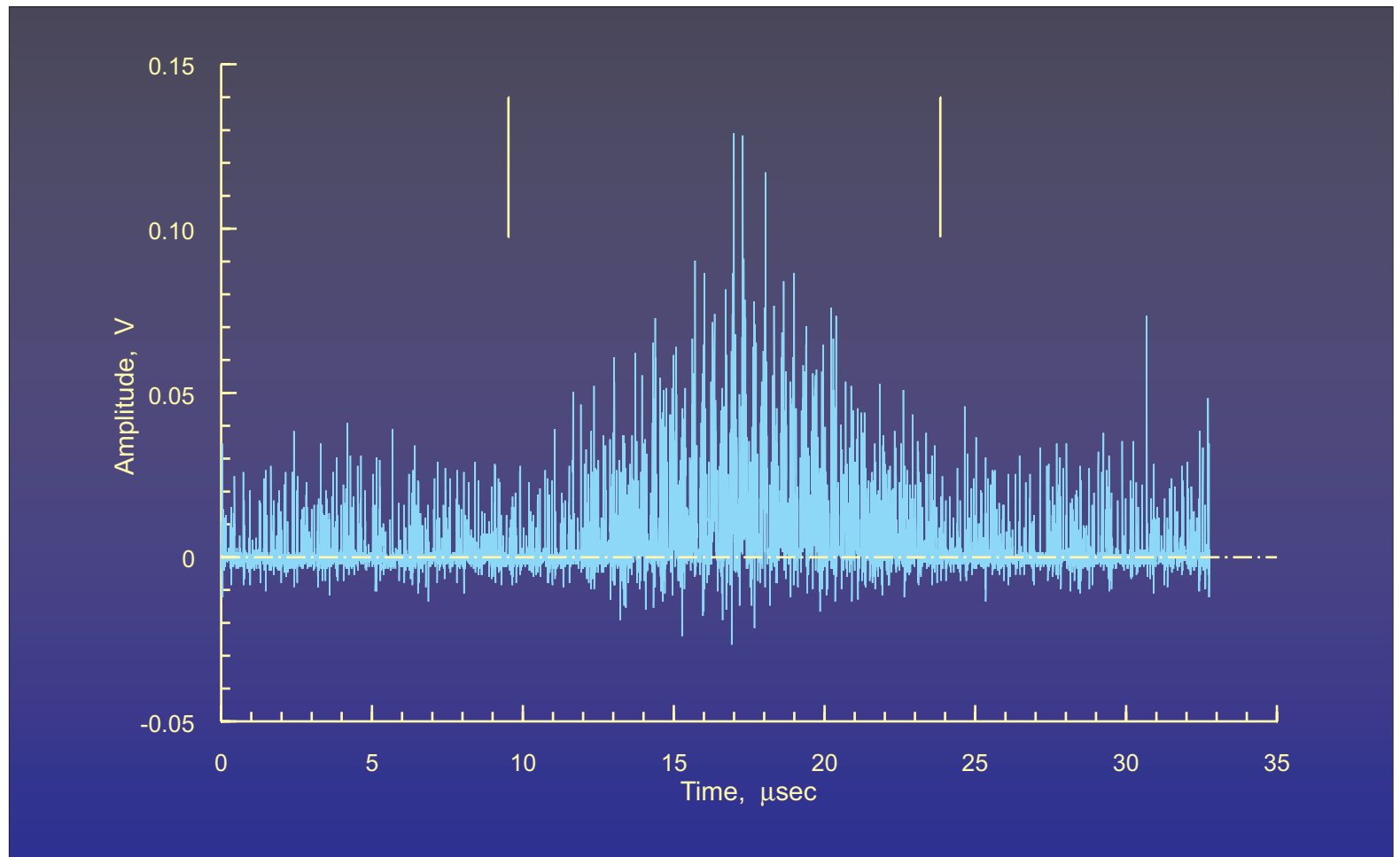

Figure 4.- Captured signal burst with added flare light from the long focal length configuration with the medium laser power setting. 


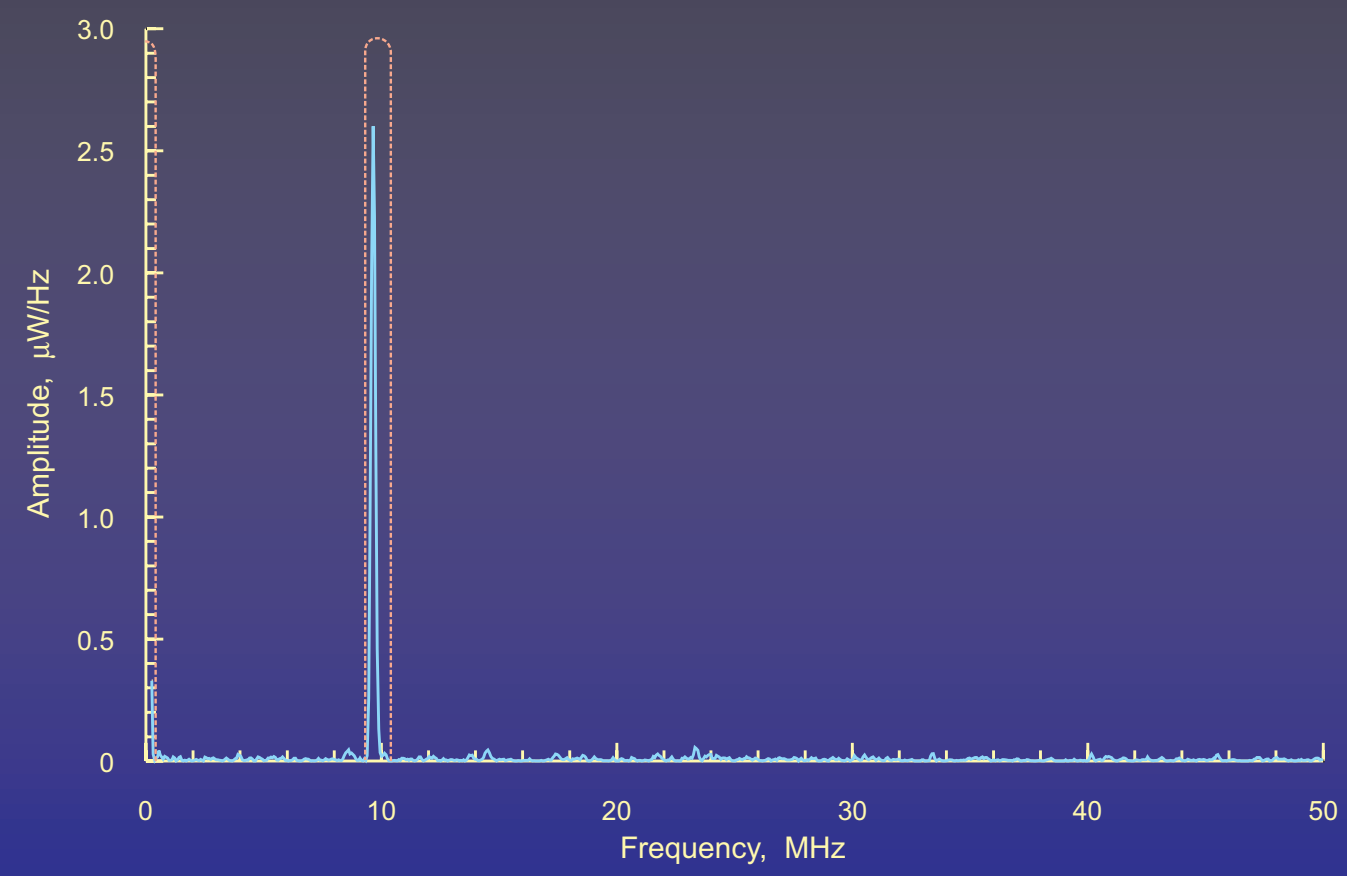

Figure 5.- Power spectra of the captured signal burst from the short focal length configuration with the low laser power setting.

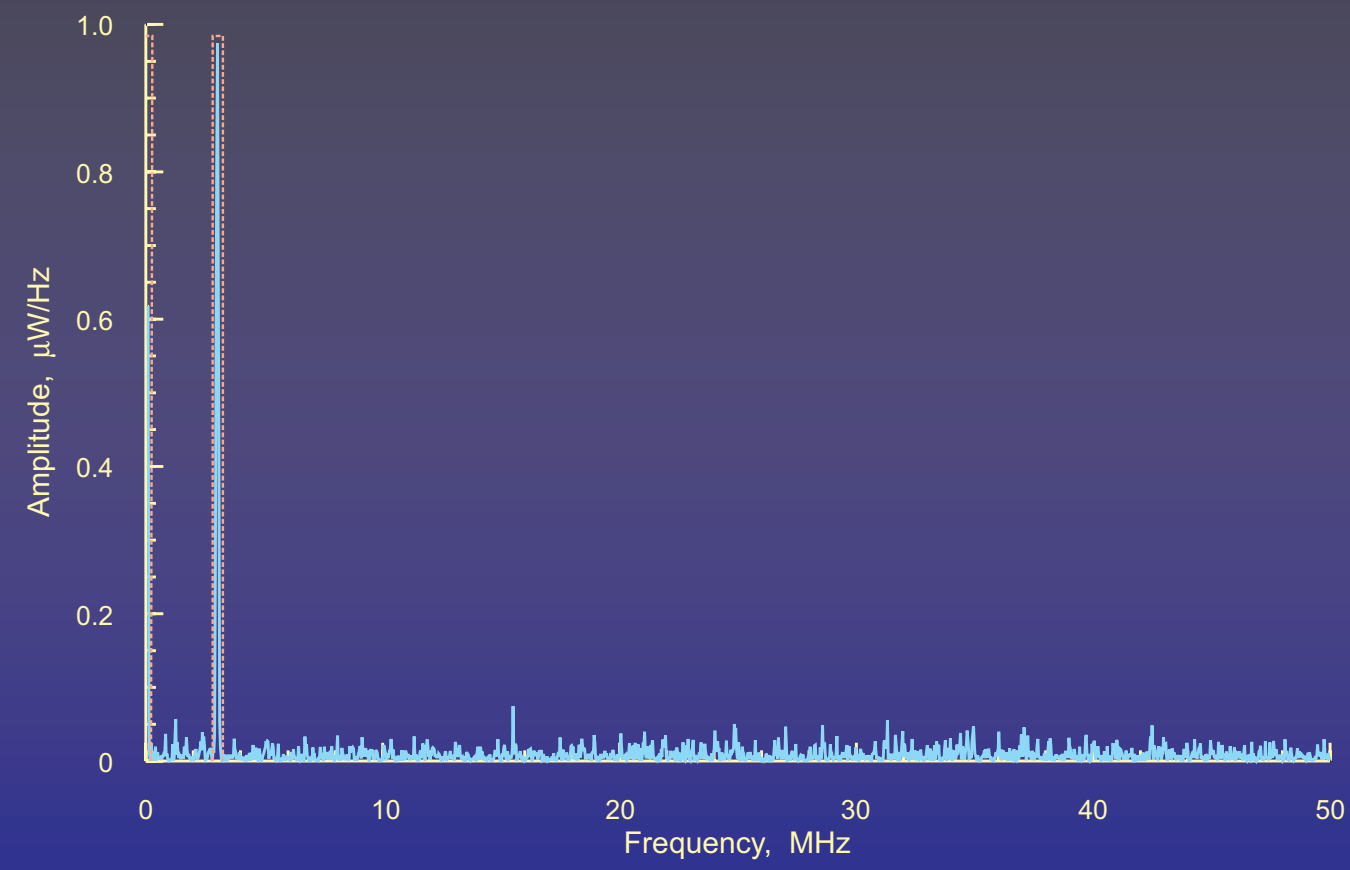

Figure 6.- Power spectra of the captured signal burst with added flare light from the long focal length configuration with the medium laser power setting. 


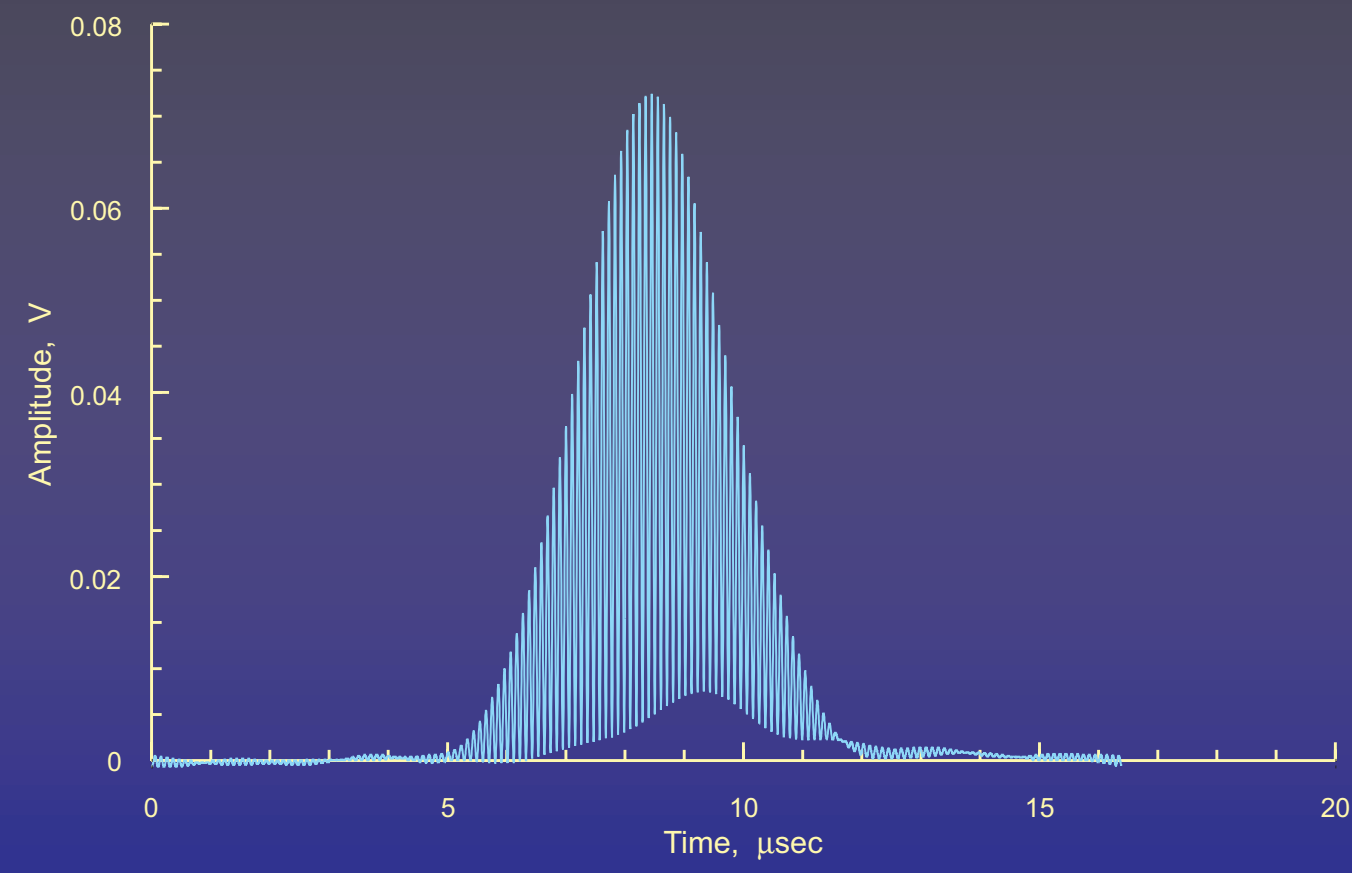

Figure 7.- Brickwall filtered signal burst from the short focal length configuration with the low laser power setting.

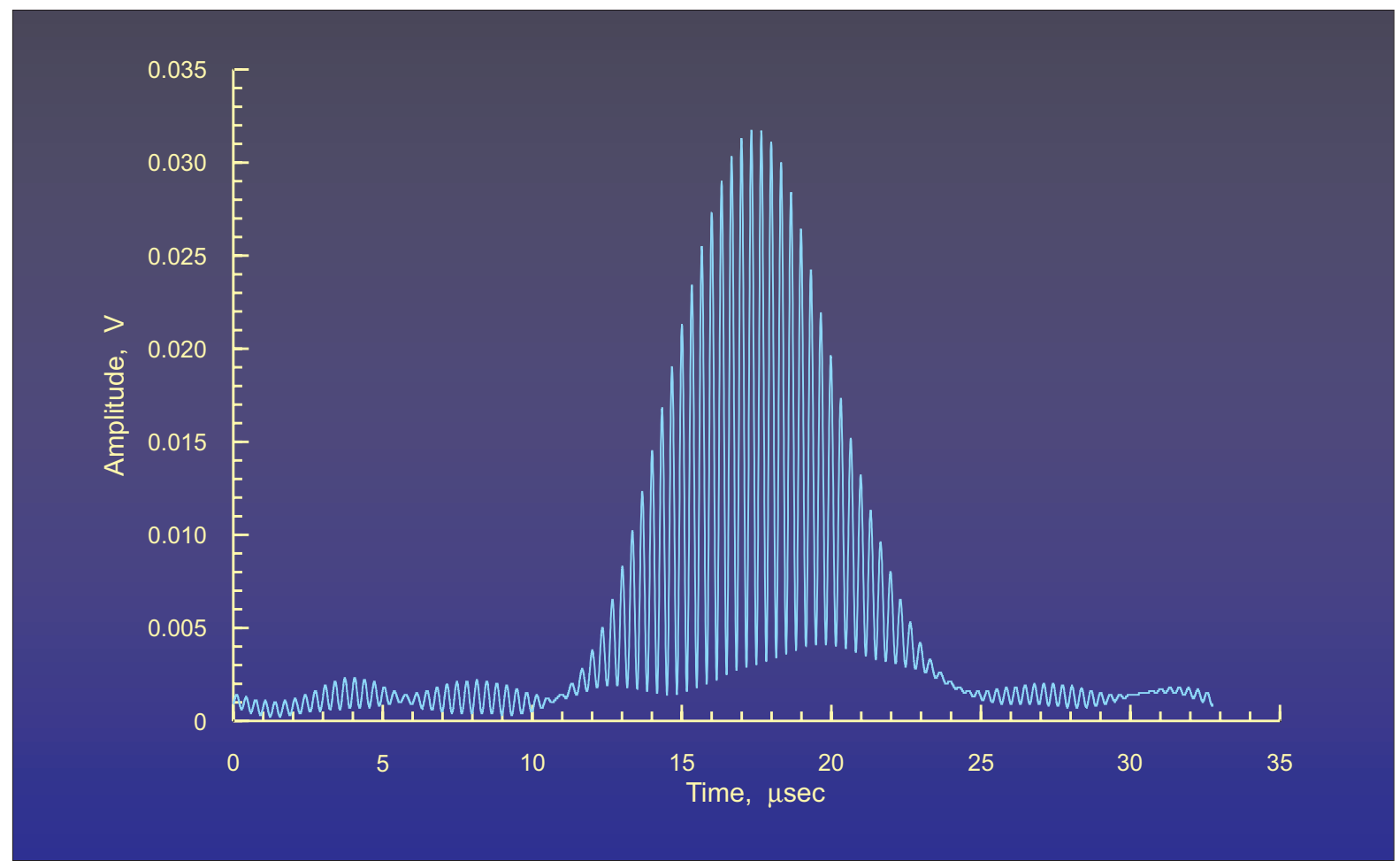

Figure 8.- Brickwall filtered signal burst with added flare light from the long focal length configuration with the medium laser power setting. 


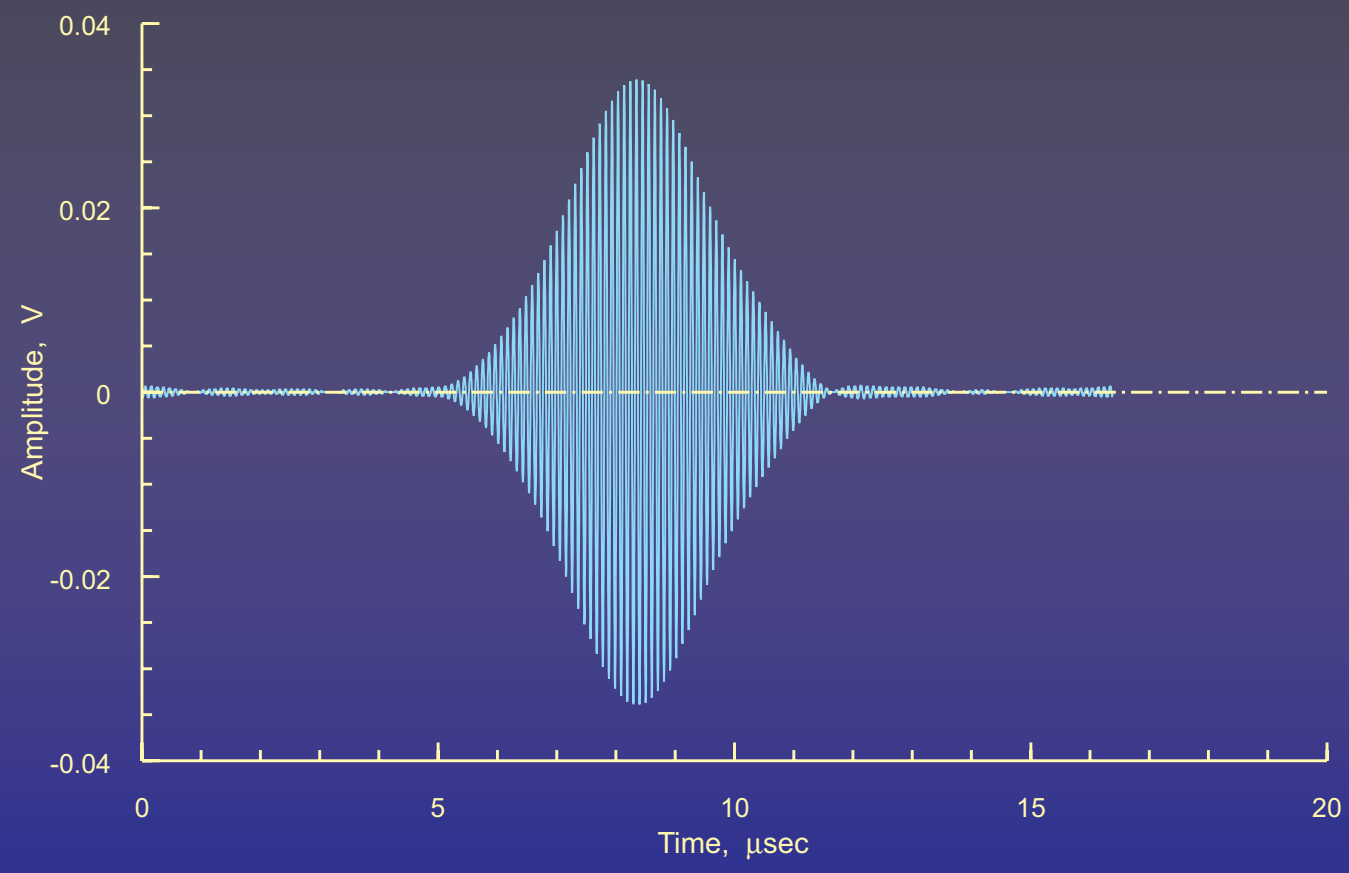

Figure 9.- Brickwall bandpass filtered signal burst from the short focal length configuration with the low laser power setting.

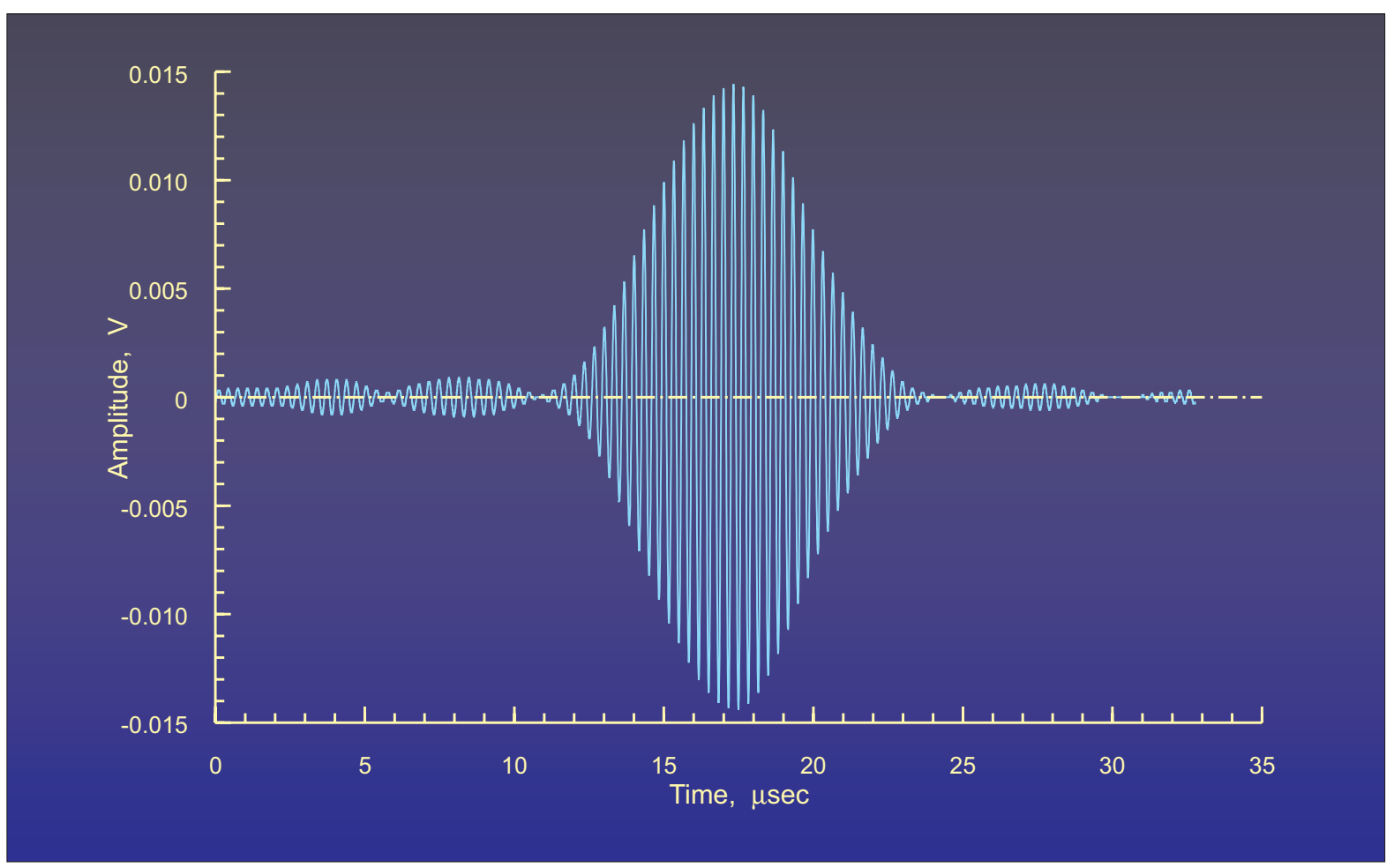

Figure 10.- Brickwall bandpass filtered signal burst with added flare light from the long focal length configuration with the medium laser power setting. 


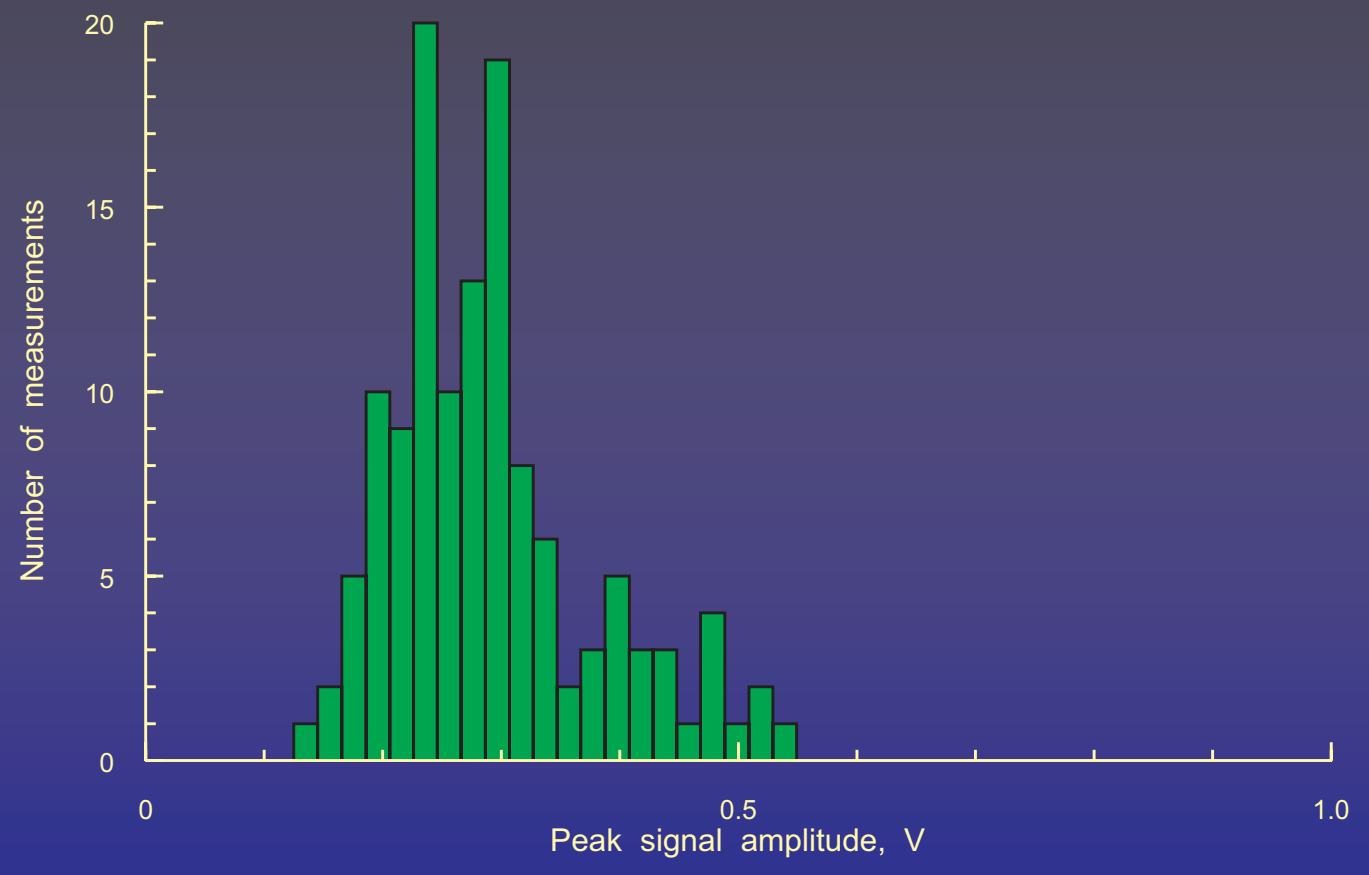

Figure 11.- Peak signal amplitude distribution from the long focal length configuration with frequency shifting.

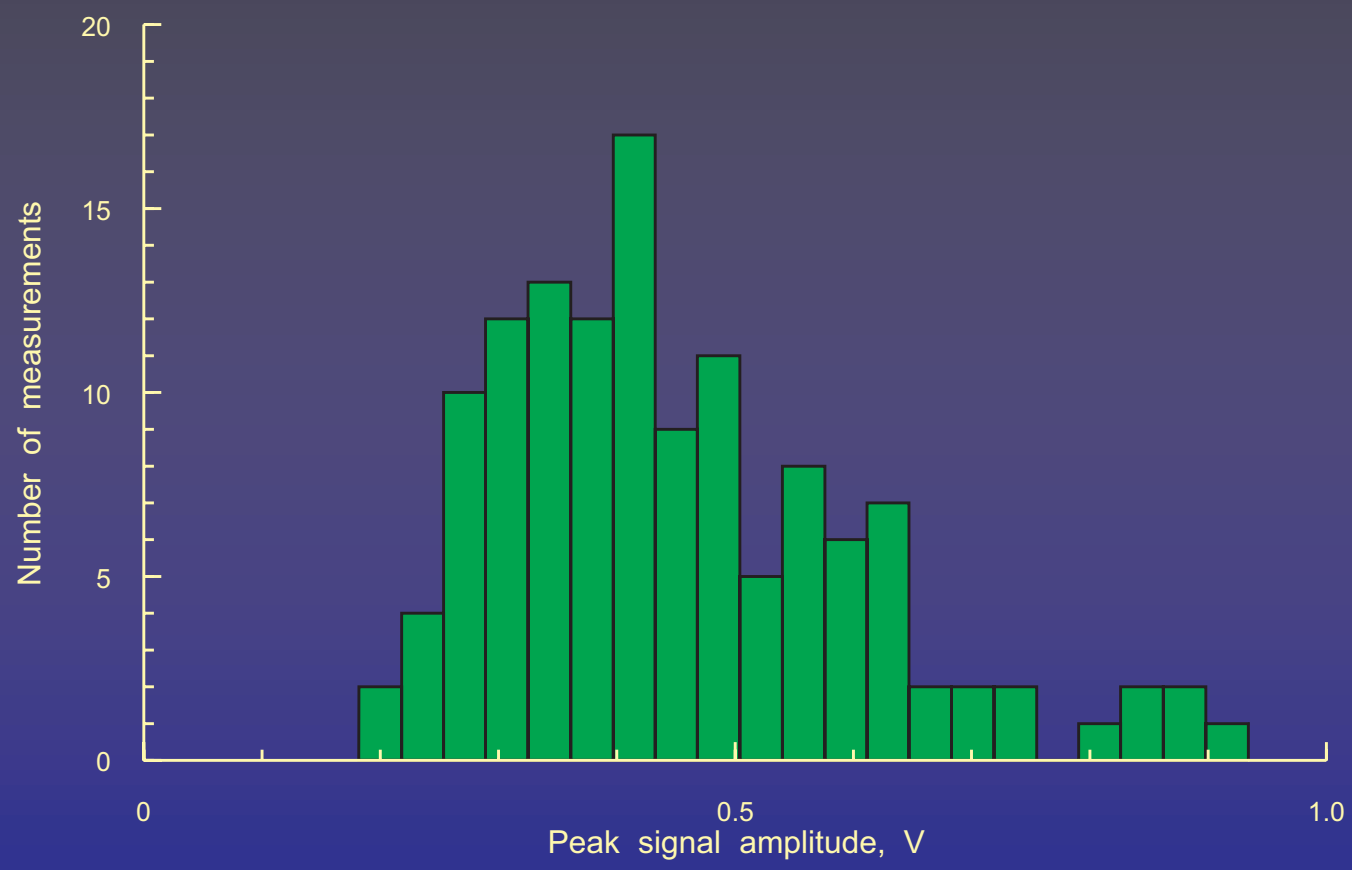

Figure 12.- Peak signal amplitude distribution from the long focal length configuration with frequency shifting and the addition of flare light. 


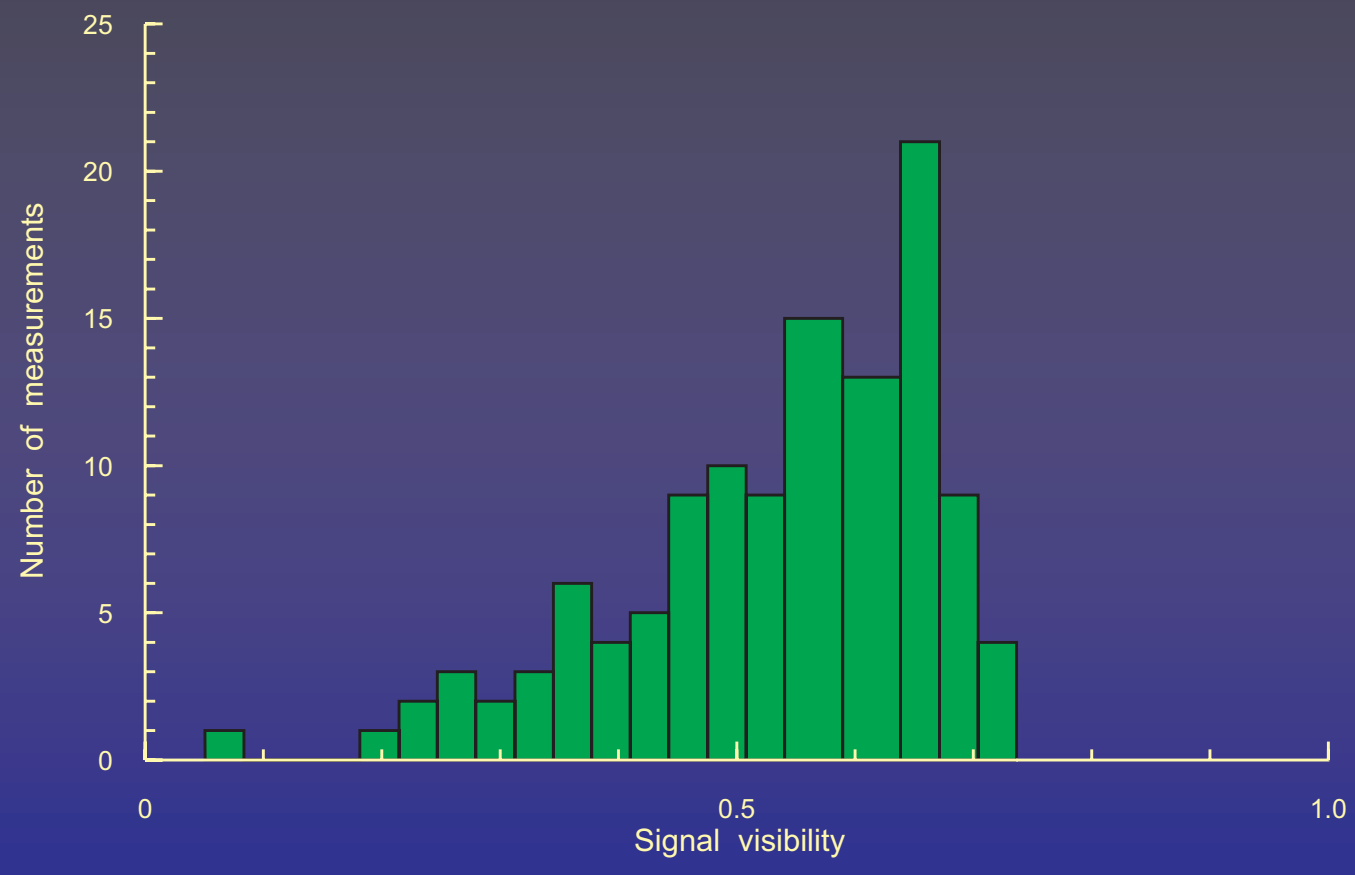

Figure 13.- Signal visibility distribution from the long focal length configuration with frequency shifting.

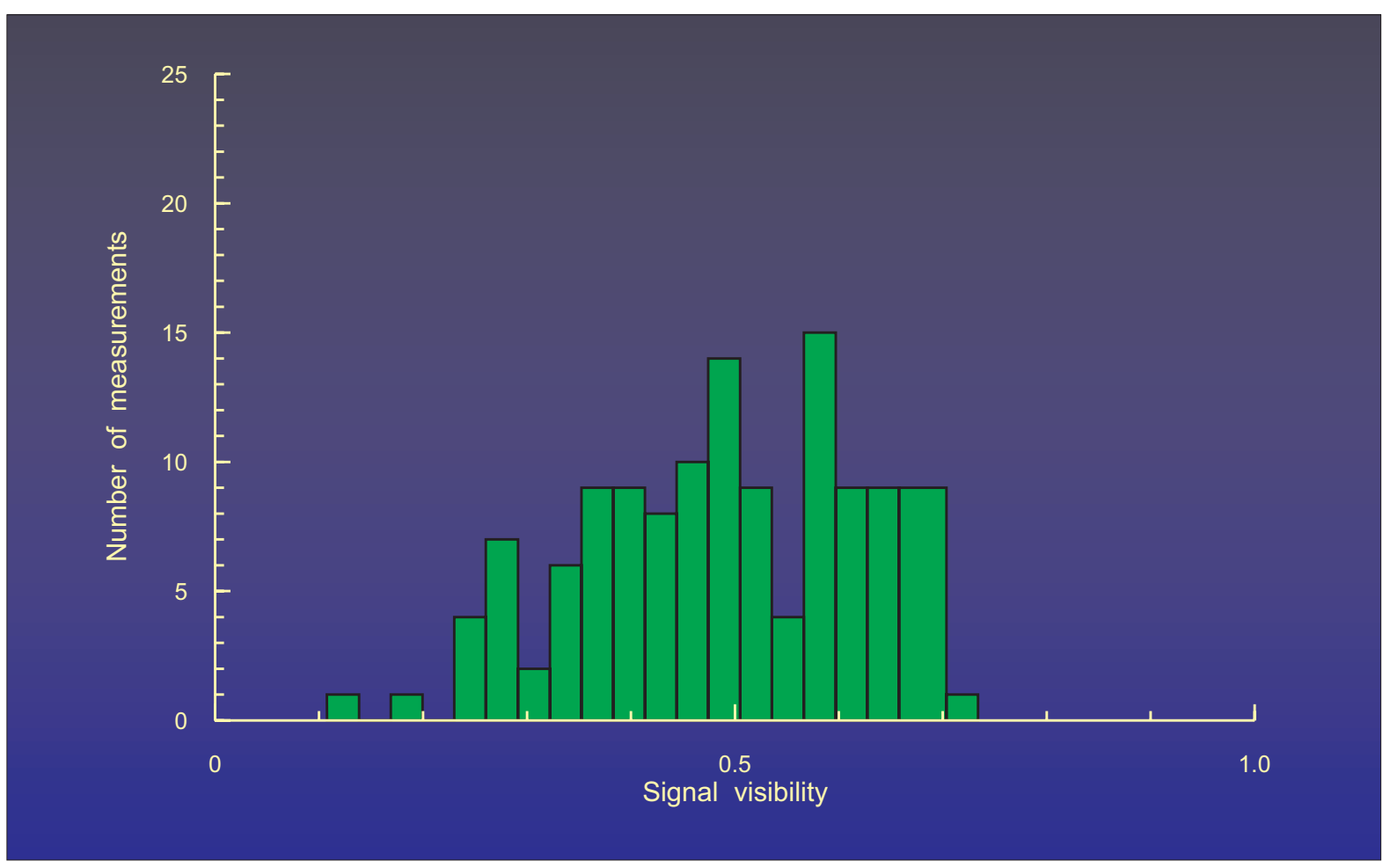

Figure 14.- Signal visibility distribution from the long focal length configuration with frequency shifting and the addition of flare light. 


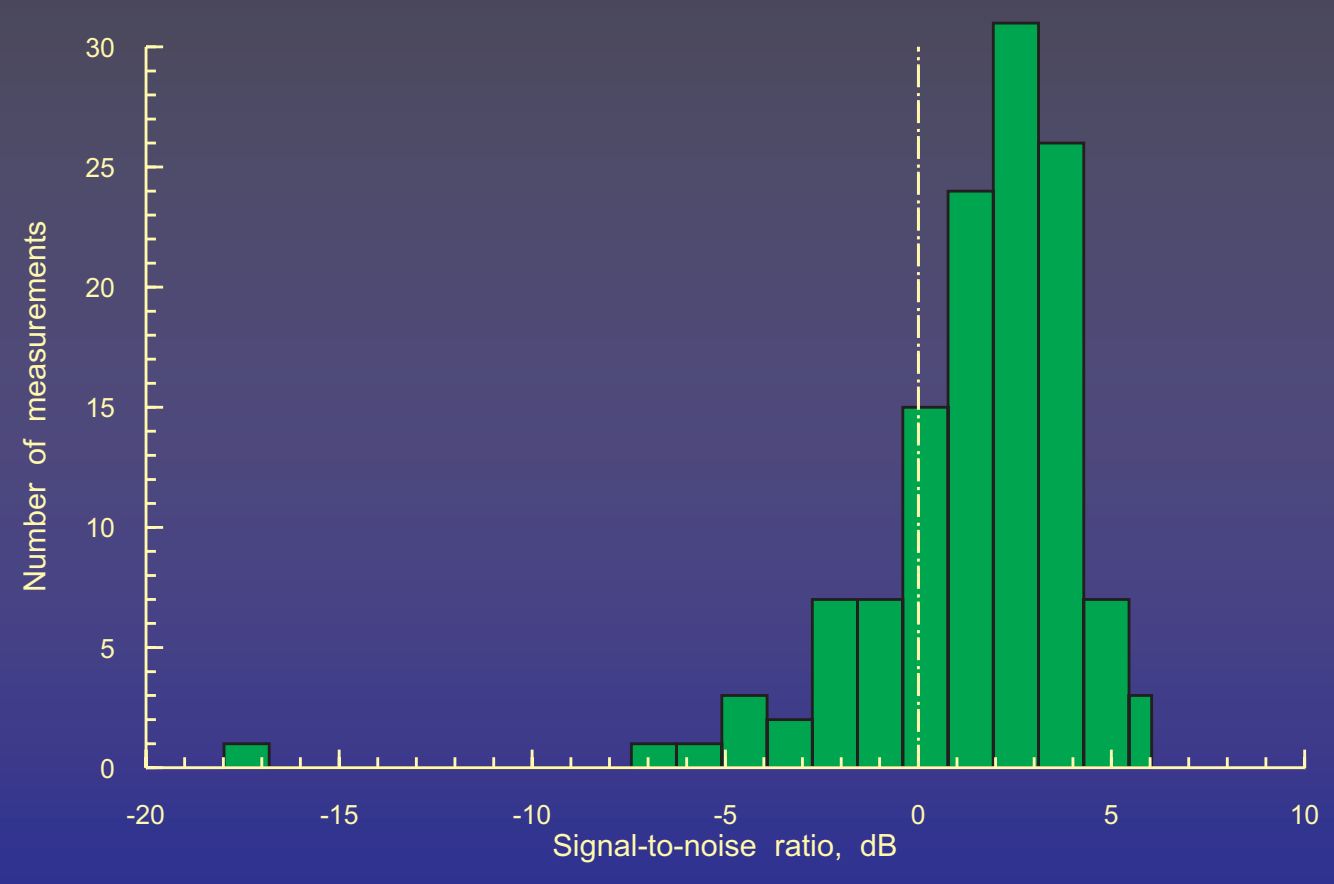

Figure 15.- Signal-to-noise ratio distribution from the long focal length configuration with frequency shifting.

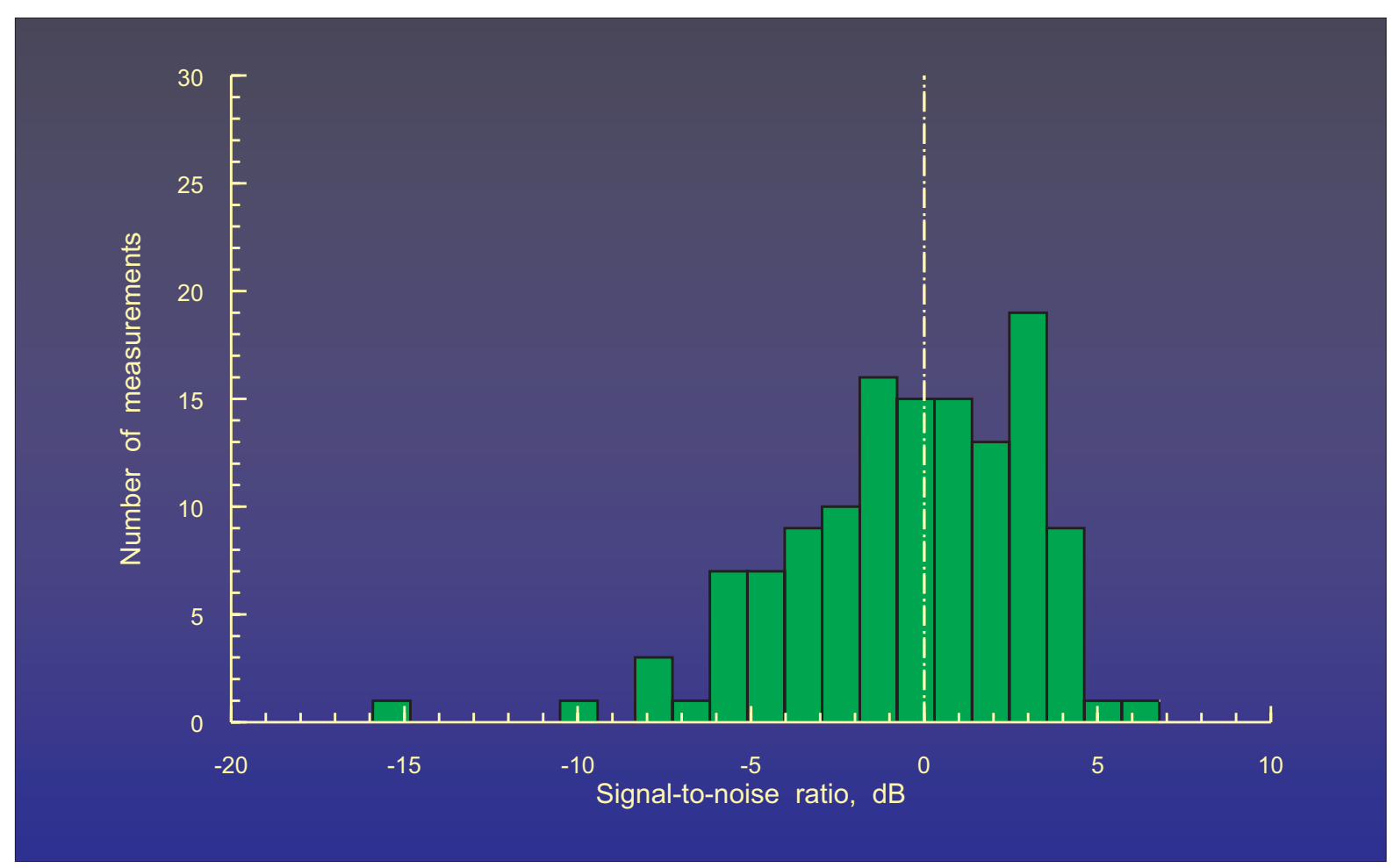

Figure 16.- Signal-to-noise ratio distribution from the long focal length configuration with frequency shifting and the addition of flare light. 


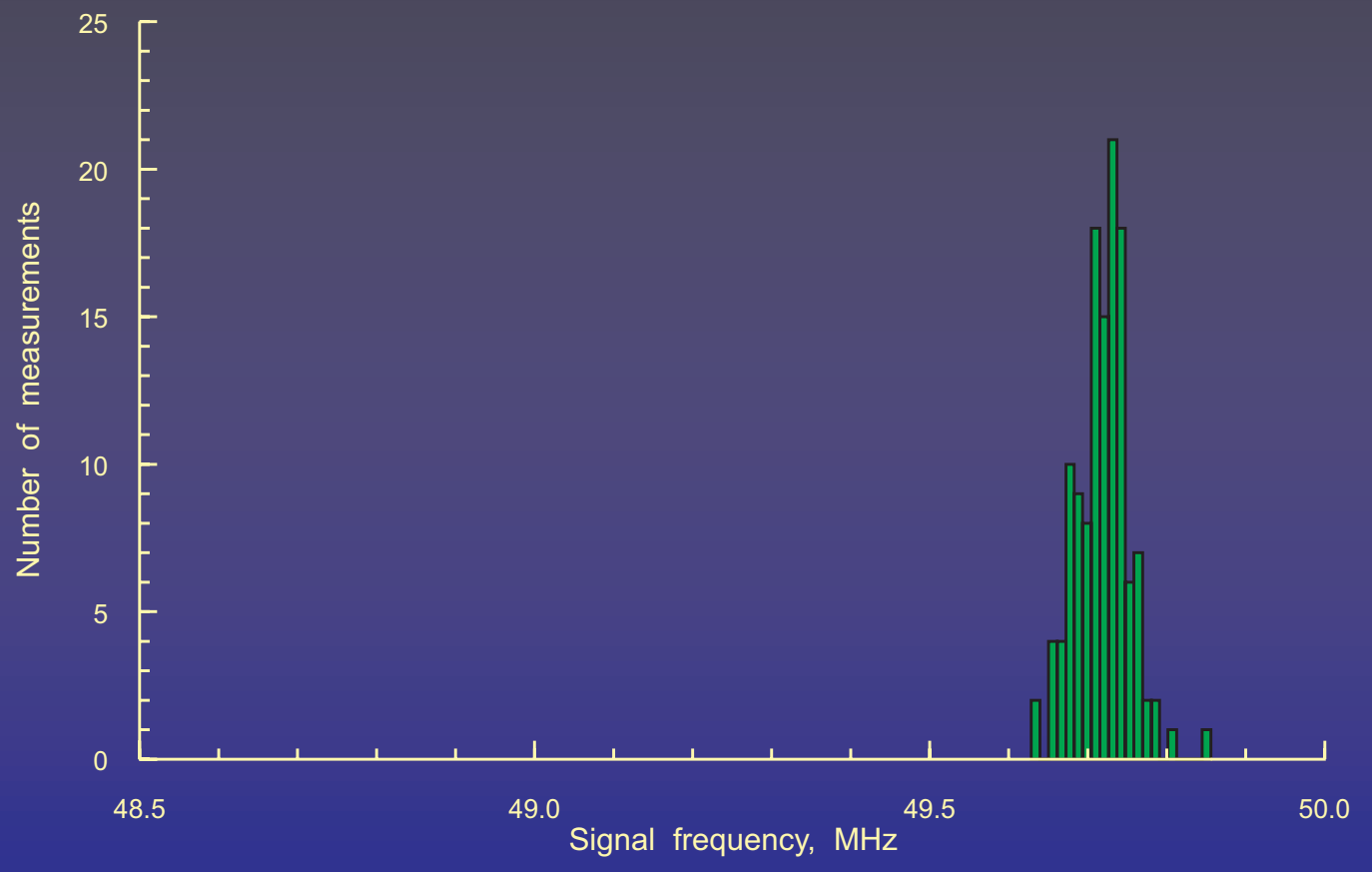

Figure 17.- Signal frequency distribution from the long focal length configuration with frequency shifting.

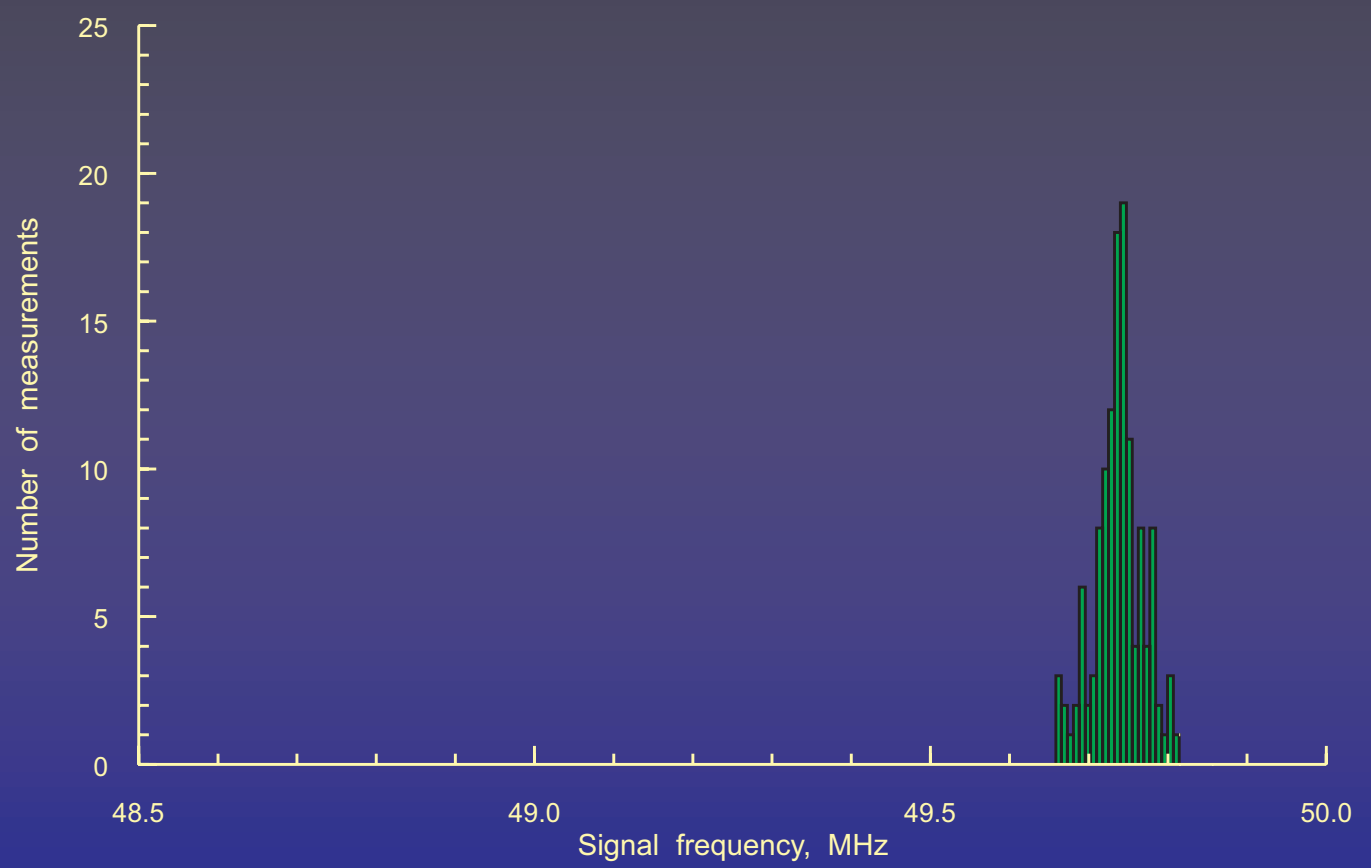

Figure 18.- Signal frequency distribution from the long focal length configuration with frequency shifting and the addition of flare light. 


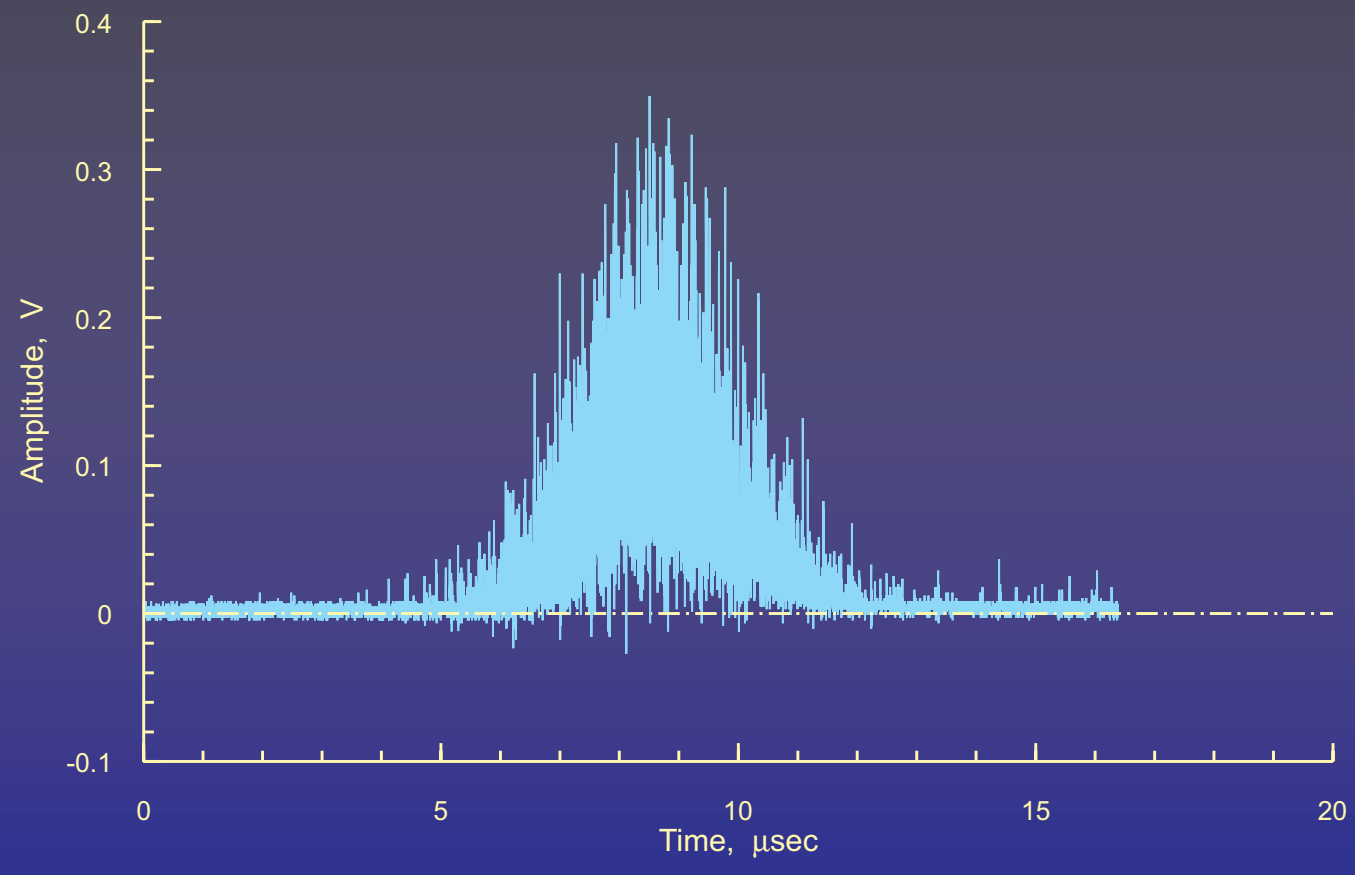

Figure 19.- An average signal burst from the long focal length configuration with frequency shifting.

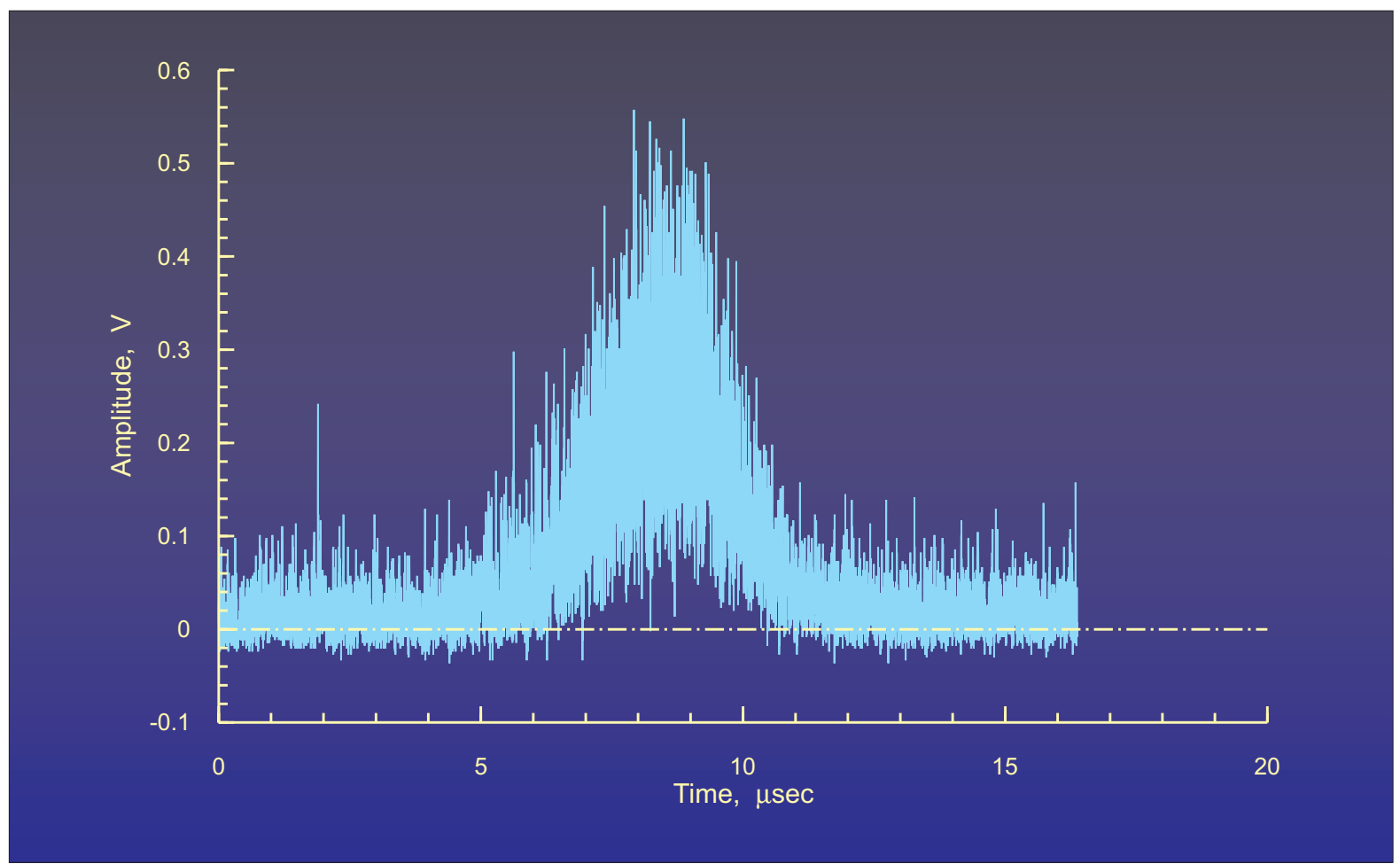

Figure 20.- An average signal burst from the long focal length configuration with frequency shifting and the addition of flare light. 